\title{
SOAR Online Course Increases Capacity for Assisting Individuals with Disabilities in the US
}

\section{Kristin Lupfer* and Jen Elder}

Substance Abuse and Mental Health Services Administration (SAMHSA) SOAR TA Center, Policy Research Associates Inc., Delmar, NY, USA

For adults with disabilities who are experiencing homelessness, chances of being approved for social security disability benefits are very low, without assistance. Assisting with the Supplemental Security Income (SSI)/Social Security Disability Insurance (SSDI) application process can be challenging for case managers who lack capacity and expertise. Training caseworkers to document disability and submit complete, highquality applications using the SSI/SSDI Outreach, Access and Recovery (SOAR) model improves efficiency and outcomes. Nationally, 65\% of applications using the SOAR model are approved, with decisions received in an average of 81 days in 2015. The SOAR Online Course was created to expand training opportunities for individuals to learn how to effectively assist with SSI/SSDI applications for individuals experiencing or at risk for homelessness. From October 1, 2014 to September 30, 2015, 1049 individuals from 49 states, Washington, DC, and Puerto Rico successfully completed the SOAR Online Course. The course is a unique public health training model; in that, it incorporates a realistic and multimodal practice SSI/SSDI application with comprehensive feedback provided by experts. Local SOAR leaders around the county are trained to facilitate and guide groups through the course. This study evaluated data on online course usage, user experience, and the translation from learning to practice for online course trainees. We found that successful course completions were most concentrated in areas that had local SOAR leaders, trainees through the online course had higher data entry rates about case outcomes in the SOAR Online Application Tracking system, and that trainees reported a high satisfaction rate with the course and comprehensive feedback. The evaluation found that key success factors for online training models include the integration of a practice case component (or other generative learning activity), support from local facilitators, and feedback and technical assistance for trainees.

\section{Keywords: homelessness, disability, social security, SSI, SSDI, mental illness, substance use disorders}

\footnotetext{
Abbreviations: OAT, Online Application Tracking; PRA, Policy Research Associates, Inc.; SAMHSA, Substance Abuse and Mental Health Services Administration; SOAR, SSI/SSDI Outreach, Access and Recovery, SSA, Social Security Administration; SSDI, Social Security Disability Insurance; SSI, Supplemental Security Income; STL, state team lead.
} 


\section{INTRODUCTION}

SSI/SSDI Outreach, Access and Recovery (SOAR) is an approach that helps states increase access to mainstream benefits for people who are experiencing or at risk of homelessness through strategic planning, training, and technical assistance.

The SOAR TA initiative evolved to address barriers to the receipt of Supplemental Security Income (SSI) and Social Security Disability Insurance (SSDI) among adults with disabilities who were experiencing homelessness. The correlation between disabilities and homelessness is high. Forty-two percent of individuals experiencing homelessness in 2013 reported having a severe mental illness or chronic substance use problem (1). For persons with mental illnesses who are homeless, SSA's disability programs can provide a steady source of income (2) and health insurance, making it possible for many to secure housing, treatment, and other needed supports $(3,4)$. Research into the social determinants of mental health has shown that improving housing can improve depression and stress (5). Additionally, individuals who have stable housing are more likely to remain in treatment and successfully complete substance use recovery programs (6).

Despite high levels of disability among people who are homeless, many who are potentially eligible never apply for benefits (7). Among those who apply, the chance of being approved for SSI/ SSDI is very low without someone taking an active role to assist with documentation of disability. Nationwide, initial approvals of SSI/SSDI applications, whether the applicant is homeless or not, average $28 \%$ (8). Denials typically result from SSA's inability to contact the individual, missed appointments, and, more generally, the lack of adequate documentation. Once an application is denied, the wait for a hearing at the administrative law judge (ALJ) level, where two-thirds of appellants are approved, averages 15 months (9).

People experiencing homelessness face many challenges when applying to SSA for disability benefits (10). These challenges generally fall into two types: those related to physical and mental health problems and the nature of homelessness and those related to more systemic issues. People who are homeless are more likely than those who have never been homeless to have serious mental illnesses alone or in combination with other disabilities, such as cognitive disorders, chronic physical health conditions, and substance use disorders (11). Disability based on a mental illness or cognitive disorder is often more difficult to document than other disabilities due to inconsistent treatment histories and difficulty in finding medical records for people who may have been treated in many places over a long time. Beyond these individual-level challenges, barriers inherent in the system of care also exacerbate a person's difficulty in applying for benefits. Chief among these is that most case managers are unable to assist people who are homeless with SSI/SSDI because they have neither the time nor the expertise. Case managers who try to help applicants are frustrated by a lack of understanding about how to make the process work more effectively.

Furthermore, the SSI/SSDI application process is timeintensive, time-sensitive, and very complex. Case managers have only 60 days from the protective filing date to navigate the complex web of requirements and complete all necessary components of the SSA packet on behalf of their clients. Case managers must then track multiple requests for information and submission of necessary information, while frequently meeting clients out in the community and often working in multiple locations in a day. For these reasons, proper training and support are critical to ensure approval. By helping increase caseworker productivity and, in turn, improving approval rates and decreasing processing times on SSI/SSDI applications, the SOAR Online Course has far-reaching benefits, which specifically address the challenges we face with an insufficient workforce and limited capacity for ongoing care.

\section{Overview of SOAR Training}

Beginning in 2001, with the support of the Substance Abuse and Mental Health Services Administration (SAMHSA), Policy Research Associates, Inc. (PRA) developed SAMHSA's Stepping Stones to Recovery manual and training curriculum, which is the basis for training in the SOAR model.

Since SOAR began, 27,226 people who were experiencing or at risk for homelessness have been approved for SSI/SSDI upon initial application through the SOAR approach. An additional 4021 persons, whose applications were denied initially, have been approved on reconsideration or appeal. Taken together, since 2006, the SOAR approach is responsible for assisting 31,247 persons to access Social Security disability benefits. The average approval rate on initial applications is $65 \%$, with decisions received in an average of 94 days in 2014 and 81 days in 2015 (12).

A significant component of the SOAR approach is training direct service staff to increase their effectiveness in completing SSI/SSDI applications. Training is currently available in two forms: in-person and online. The in-person, 2-day training is delivered by local SOAR trainers who attended a SAMHSA-sponsored, PRA-provided, train-the-trainer (TTT) program between 2004 and 2013. Since 2004, PRA has trained 1004 trainers who conducted 1318 SOAR trainings.

Despite significant resources dedicated to the in-person training model, an external evaluation of SOAR found that only $13 \%$ of trainees who received the in-person training were actually assisting with applications post-training (13). This corresponds with other research documenting that in-person trainings result in lower student performance compared with online training (14). We know that some proportion of those trainees never assisted an individual experiencing homelessness with SSI/SSDI because they were intimidated by the idea of doing their first application. The SOAR Online Course addresses this particular barrier and opens up new opportunities to build system capacity to end homelessness in states and communities.

\section{Transition to the SOAR Online Course}

The SOAR online training content and website were developed between 2010 and 2013. Pilot and beta testing of the training extended throughout 2014, and course content was revised based on feedback. Specific improvements included clarifying terms and fixing typos in course articles, re-ordering the presentation of information, and addressing technical difficulties. The course was released to the public in October 2014 after final review by SAMHSA and SSA staff and in conjunction with the start of a 5-year SAMHSA contract for the SOAR TA center (base year and 
4 option years). While the contract year began from September 15,2014 , the authors evaluated activity from October 1, 2014 to September 30, 2015, with the sole exception of the trainee survey, explained below.

The SOAR Online Course consists of seven classes, each of which has a series of articles, videos, short quizzes, and a practice case. The course trains individuals in the core elements of completing SSI/SSDI applications using the SOAR model and includes a practice case. The practice case provides an opportunity for case managers to apply what they have learned by completing an SSI/SSDI application packet for a fictitious applicant. Video interviews, medical records, and progress notes provide the information needed to complete SSA forms and write a medical summary report (MSR). The packet is submitted to the SOAR TA center for review, and each application receives extensive written feedback. Upon approval, the participant receives a certificate of completion and 16 continuing education units (CEUs) from the National Association of Social Workers (NASW). We estimate that it takes about $16 \mathrm{~h}$ to complete the entire course with participants working at their own pace. However, we encourage students to complete the course within 30 days to retain the information learned and get connected sooner to local SOAR initiatives.

Studies show that students retain more information when they involve active learning, such as generative activities, whereby they must apply the information learned, self-assessment and reflection, and increased time to review and re-read course materials (14-17). The course and practice case allows trainees the flexibility to complete the training at their own pace and review sections or return to it whenever they need to brush up on a particular topic. While the addition of a practice case may result in fewer people completing the online training than those who attended in-person trainings, this active learning approach will better prepare trainees to assist with SSI/SSDI applications.

The online training brings standardization to the curriculum and ensures that all SOAR-trained providers have received the same basic training. Course coordinators review the online curriculum monthly and update content according to changes in SSA regulations. As a result, trainees receive the most up-to-date information on the disability process. By ensuring that the training is always up-to-date and providing timely, comprehensive feedback and technical assistance to trainees, the course is designed to attract and retain trainees based on guidance provided by Ballew et al. (18).

SSI/SSDI Outreach, Access and Recovery community leaders and state team leads (STLs) can follow-up with tailored 1-day trainings to provide information specific to their state's SOAR process. Some leads also form facilitated online training cohorts, whereby they guide a group of participants through the course. This can include weekly check-in calls to assess trainee progress and understanding. A meta-analysis of online training programs found that student performance in online training settings was highest in courses that blended online and in-person training (14).

The online training is accessible wherever there is an Internet connection, thus expanding the impact of SOAR to rural and other communities that have not had previous access to trainings. Ballew et al. (18) found that online trainings increase access and flexibility for trainees but noted that lack of reliable Internet service or updated technology is still a barrier in fully implementing online training in all areas.

While the literature documents the need for increased interactivity, beyond a study evaluating training utilizing virtual three-dimensional environments (19), there are no evaluations analyzing the use of mock applications in an online setting to increase skills for social service providers. This evaluation intends to fill this gap and analyze the effectiveness of incorporating a realistic and multimodal practice case application with comprehensive feedback provided by experts, while also facilitating the completion of the online training through live guided cohorts.

\section{EVALUATION METHODS}

Statistics used for the evaluation were gathered from October 1, 2014 through September 30, 2015 (base year). We analyzed the activity and outcomes from all course users who either completed the course or enrolled in the course during this time frame ( $n=4477$ users).

In the base year, 1140 individuals completed the course and submitted documents to the SOAR TA center for review; of these individuals, 1049 successfully passed the course. A majority, 645 trainees (56.6\%), passed on the first submission and 404 trainees (35.4\%) passed on a second, revised submission. Eleven individuals were unable to successfully complete the course, and packets for 80 individuals were either incomplete, under review, or had been reviewed with revisions requested and were still pending. A further 3337 individuals enrolled in the course during the base year but did not complete the training within the time period under evaluation.

For the practice case, participants are randomly assigned at equal rates a male or female case study. There was no significant difference in the pass rates between those who received the male or female case study. As a result, this evaluation did not explore differences in the case studies themselves that may have impacted course outcomes.

We used a multipronged approach to evaluate quantitative data on course usage and the translation of learning to practice, and qualitative data about user experience. To gather the course usage data, we utilized Google Analytics for website and course page use, YouTube Analytics to assess course video use, and mapped course completion rates from the SOAR database that tracks course progress and results. We sought to assess: (1) the number of people who looked at the course website compared to enrollment statistics, (2) the geographic reach of the course, (3) differences in completion rates for individuals who have a nearby SOAR Lead compared to areas without a SOAR Lead, (4) average length of time to completion from course enrollment to practice case submission, and (5) utilization of course materials.

To gather qualitative data about user experience, we delivered surveys via SurveyMonkey to gather information about the characteristics of who is completing the course and responses about course content. The survey was sent to the individuals who passed the course during the dates of the FY2015 contract year for the SAMHSA SOAR TA center, September 15, 2014-September 14, 2015. The survey link was sent to 863 people who had completed the review process for the practice case and successfully passed the course in that time frame. The evaluation survey was then 
sent to everyone who passed or failed ongoing from September 15, 2015 to track feedback for FY2016. The survey responses are anonymous, so we are unable to identify the people who received and potentially responded to the survey in the 2 weeks left out of the evaluation period (September 15-30, 2015) to add them in and the 2 weeks prior (September 15-30, 2014) to remove them. This partially explains the discrepancies in our course completion totals and survey totals. The balance can be explained by the fact that after participants submit their practice case applications for review (i.e., complete the course), they may be required to revise and resubmit forms. The time required for resubmission and additional review can cause their original submission and pass/ fail dates to fall into two different fiscal years.

The survey asked respondents to provide detail about their background with eight multiple choice questions (agency type, role, funding source, current application assistance outcomes, and cohort participation). Respondents were asked to measure their agreement on a 4-point scale with 19 statements relating to the online course (content comprehension, course content, and the practice case component). Two additional questions asked respondents to rate their satisfaction on a 5-point scale regarding information, organization, and presentation of course materials. Finally, the survey included three open-ended questions about likes, dislikes, and suggested improvements to the course.

Further, we cross-referenced those who successfully completed the course during the evaluation period with SOAR Online Application Tracking (OAT) system outcomes to compare SSI/ SSDI application outcomes between those who received the online training and those who took the in-person training. The SOAR OAT program is online, HIPAA-compliant, and accessible from any web-enabled device. It is free to access, user-friendly, and asks as few questions as possible to minimize data entry burden. While not all SOAR programs utilize the OAT system to track outcomes, data were sufficient to analyze trends between the training methods.

These evaluation methods were undertaken to address five research questions:

1. What types of caseworkers are utilizing the SOAR Online Course?

2. What benefits and barriers can we identify by using a practice case component in the online training?

3. Does facilitated training (i.e., cohorts and follow-up) increase participation in the training?

4. Does online training increase the quality and quantity of submitted SSI/SSDI applications compared to in-person training?

5. How can these results be expanded into other areas of online training for social services?

\section{EVALUATION FINDINGS}

\section{Enrollment Statistics and Attrition during Base Year}

The evaluation found high attrition rates in the SOAR Online Course, with only $25.43 \%$ of total enrollees completing the course during the base year. This is consistent with previous studies that found high attrition rates in free, non-facilitated online courses due to a number of people enrolling in the course as "window shoppers," without intentions of completing the training (20). In addition, lack of organizational support during the training and lack of relevance to a learner's job have been found to predict increased course dropout rates (21). This current study did not evaluate the reasons behind the high attrition rate for the SOAR Online Course enrollees, and as Stone (20) notes, future research is needed in this area.

\section{Course Usage Mapping and Correlation with SOAR Leaders}

To evaluate course reach and to examine possible correlations between successful course completions and the presence of a local SOAR leader, we created a map with three sets of data: number of enrolled online course users by state, successful course completions by zip code, and the location of a local SOAR leader by city and state. The information about course enrollees and completions was pulled from an internal database for the SOAR Online Course. Location information for SOAR leaders was gathered from an internal database of individuals who attended a 3-day SOAR Leadership Academy, sponsored by SAMHSA, in the 2-year period before the end of the base year. This time frame allowed us to map any potential impact SOAR leaders have on usage of the SOAR Online Course.

Figure 1 shows the distribution of successful completions by state through shading gradients, the number of successful course completions by zip code using blue to red heat mapping, and the locations of SOAR leaders by city using icons. Alaska and Puerto Rico are not pictured, but each had three successful course completions in the base year and do not have any SOAR leaders. Hawaii was excluded from Figure 1, as it also had an insignificant amount of SOAR Online Course activity during the evaluation period.

Through mapping the course usage, we tested our hypothesis that the SOAR Online Course would expand the training to new areas around the country. All states had enrollees in the course and trainees from 49 states, the District of Columbia, and Puerto Rico successfully completed the course during the base year. Ohio did not have any providers complete the SOAR Online Course in this timeframe.

The significant finding in this map is that successful course completions are clustered in the areas that have local SOAR leaders, particularly in the areas with high concentrations of completions. Through the Leadership Academy, SOAR leaders are trained on how to facilitate groups through the course and support trainees, and this may be a key contributing factor to this finding. Evaluating the causal link between these factors was outside the scope of the current study, though future research into this area would be beneficial.

\section{Days to Successful Completion}

To determine the length of time to complete the course, we calculated the days from course enrollment to the date the course packet was submitted. We matched the days to completion with the role the trainee most identified with during 


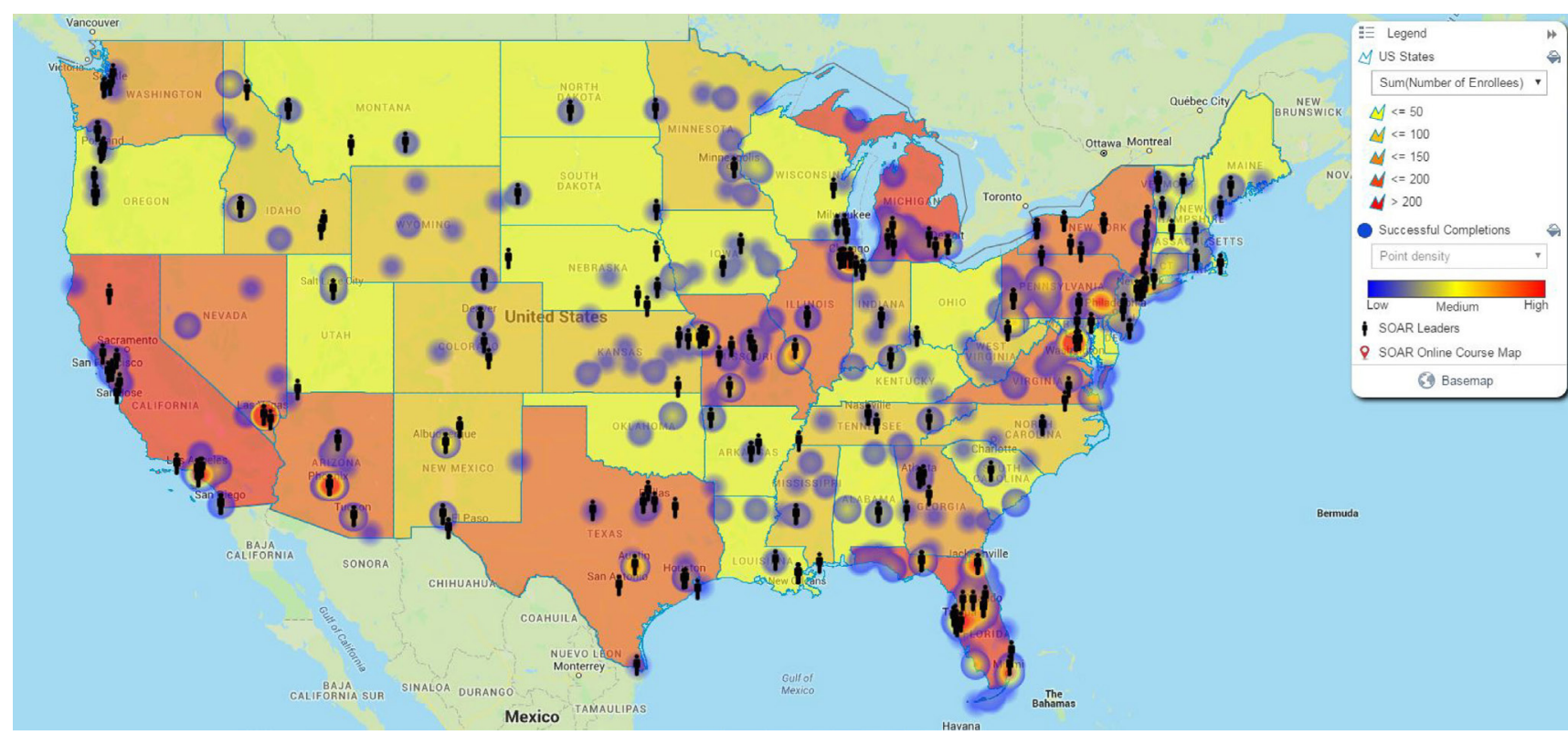

FIGURE 1 | SOAR Online Course enrollments, completions, and locations of SOAR Leads.

their course registration: agency director, case manager, local SOAR lead, peer specialist, SOAR state team lead, or personal use. The latter category indicates that the trainee was taking the course to help himself or herself or a friend/family member.

We also evaluated who was successfully completing the SOAR Online Course, by role, during the base year. The majority of successful completions (52\%) were by those who self-identified as a case manager. State, local, and agency leads represent a minority of course completions and may be reflective of supervisors taking the program before introducing it to their staff. Of note, 360 trainees $(34.3 \%)$ left the role blank.

As Table 1 illustrates, SOAR Online Course trainees take approximately 44 days to complete the training, and case managers represent over half of successful completions.

\section{Utilization of Course Materials}

Each of the two practice cases in the SOAR Online Course has three video interviews with the mock applicant. Watching these videos in full is a key to successful completion of the training. To evaluate the utilization of the course videos, we used YouTube Analytics gathered from the base year to determine the average percentage that each course video was viewed. Table 2 presents these findings.

A Pearson product-moment correlation was run to determine the relationship between the length of the practice case videos and the percentage of the video watched. The data showed no violation of normality, linearity, or homoscedasticity, after one outlier (male video 1) was removed prior to calculations. The male actor in video \#1 spoke quickly and with a strong accent, which may have led to difficulties in understanding him, requiring repeated
TABLE 1 | Average days to completion of the SOAR Online Course by role.

\begin{tabular}{lccc}
\hline Role & $\begin{array}{c}\text { Number of } \\
\text { completions }\end{array}$ & $\begin{array}{c}\text { Percentage of total } \\
\text { successful completions }\end{array}$ & $\begin{array}{c}\text { Average days } \\
\text { to completion }\end{array}$ \\
\hline $\begin{array}{l}\text { Agency director } \\
\text { Case manager }\end{array}$ & 61 & 6 & 43.1 \\
Local SOAR & 544 & 32 & 45.7 \\
lead & 29 & 3 & 44.2 \\
$\begin{array}{l}\text { Peer specialist } \\
\text { SOAR state }\end{array}$ & 36 & $<1$ & 35.6 \\
team lead & 5 & 1 & 78 \\
$\begin{array}{l}\text { Personal use } \\
\text { Other (role left }\end{array}$ & 14 & 34 & 55.8 \\
blank) & 360 & 100 & 41.7 \\
Grand total & 1049 & & 44.1
\end{tabular}

viewing. This may account for the high percentage of views for the male video \# 1 compared to the others.

There was a strong, negative correlation between length of video and percentage viewed, which was statistically significant $(r=-0.976, n=5, p<0.005)$, resulting in a finding that an increase in video length is correlated with a decrease in percentage of the video viewed.

\section{Qualitative Findings about User Experience}

In October 2015, the SOAR TA center surveyed individuals who successfully completed the course during the base year. Surveys were delivered via SurveyMonkey to 863 individuals to gather background information and characteristics of 
TABLE 2 | Data and analysis of the SOAR Online Course YouTube videos.

\begin{tabular}{|c|c|c|c|}
\hline Video segment & $\begin{array}{l}\text { Full duration } \\
\text { of video }\end{array}$ & $\begin{array}{l}\text { Average percentage } \\
\text { of video viewed }\end{array}$ & $\begin{array}{c}\text { Number of } \\
\text { viewers }\end{array}$ \\
\hline Male video 1 & $5: 15$ & 102.47 & 2276 \\
\hline Male video 2 & $3: 50$ & 93.71 & 1434 \\
\hline Male video 3 & $4: 50$ & 92.2 & 1087 \\
\hline Female video 1 & $8: 29$ & 88.34 & 2127 \\
\hline Female video 2 & $6: 22$ & 89.16 & 1433 \\
\hline \multirow[t]{2}{*}{ Female video 3} & $9: 44$ & 85.91 & 1198 \\
\hline & & Video length & $\begin{array}{l}\text { Percent } \\
\text { viewed }\end{array}$ \\
\hline \multicolumn{4}{|l|}{ Statistics } \\
\hline Mean & & 0:06:39 & 89.8640 \\
\hline SEM & & 0:01:05 & 1.39106 \\
\hline Median & & 0:06:22 & 89.1600 \\
\hline Mode & & $0: 03: 50^{a}$ & $85.91^{a}$ \\
\hline SD & & $0: 02: 27$ & 3.11050 \\
\hline Variance & & $21,764.000$ & 9.675 \\
\hline Range & & 0:05:54 & 7.80 \\
\hline Minimum & & 0:03:50 & 85.91 \\
\hline Maximum & & $0: 09: 44$ & 93.71 \\
\hline \multirow[t]{3}{*}{ Percentiles } & 25 & 0:04:20 & 87.1250 \\
\hline & 50 & 0:06:22 & 89.1600 \\
\hline & 75 & 0:09:06 & 92.9550 \\
\hline \multicolumn{4}{|c|}{ Pearson product-moment correlation } \\
\hline \multirow[t]{3}{*}{ Video length } & $\begin{array}{l}\text { Pearson } \\
\text { correlation }\end{array}$ & 1 & $-0.976^{b}$ \\
\hline & Sig. (2-tailed) & & 0.004 \\
\hline & N & 5 & 5 \\
\hline \multirow[t]{3}{*}{ Percent viewed } & $\begin{array}{l}\text { Pearson } \\
\text { correlation }\end{array}$ & $-0.976^{b}$ & 1 \\
\hline & Sig. (2-tailed) & 0.004 & \\
\hline & N & 5 & 5 \\
\hline
\end{tabular}

aultiple modes exist. The smallest value is shown.

${ }^{b}$ Correlation is significant at the 0.01 level (2-tailed).

Coefficient of determination $\left(r^{2}\right)=0.953$.

participants completing the course and quantitative and qualitative responses to the course content and self-assessed comprehension. One hundred and thirty-six people responded to the survey (15.76\% response rate), and the results are below in Table 3.

Table 4 displays the results of the SOAR Online Course evaluation portion of the survey. The majority, $96 \%$ of respondents, agreed or strongly agreed with the statement: "Overall, I feel this training will help me do a better job assisting individuals with SSI/SSDI applications." Similarly, 96\% of respondents were satisfied or very satisfied with the information provided in the SOAR Online Course. Note that for this portion of the survey, $n=122$, as 14 participants did not respond to these questions.

A majority of respondents (63\%) indicated that prior to completing the SOAR Online Course they had not assisted with any SSI/SSDI applications over the past year, while $23 \%$ indicated that they assisted with less than a single application per month. Over half of respondents who assisted with SSI/SSDI applications had less than a $25 \%$ approval rate prior to taking the training, with almost a quarter of respondents having a $0 \%$ approval
TABLE 3 | Respondent characteristics from the survey of base year trainees.

\begin{tabular}{lrr}
\hline & $\boldsymbol{n}$ & $\%$ \\
\hline Agency & 45 & 33 \\
Community mental health agency & 39 & 29 \\
Homeless service agency & 4 & 3 \\
Veteran services agency & 9 & 7 \\
State agency & 39 & 29 \\
Other & & \\
Position & 51 & 38 \\
Case manager & 10 & 7 \\
Outreach worker & 1 & 1 \\
Shelter worker & 13 & 17 \\
Benefits specialist & 23 & 28 \\
Program coordinator/supervisor & 38 & \\
Other & & \\
Other characteristics & 46 & 35 \\
Participated in the SOAR Online Course as part of a & & \\
facilitated local training group/cohort & 48 & 37 \\
Planning to attend a local SOAR Fundamentals refresher & 50 & 37 \\
training after the online course & & \\
Assisted adults with SSI/SSDI applications in the past year & 53 \\
\hline
\end{tabular}

rate. Notably, after completing the SOAR Online Course $94 \%$ of respondents agreed with the statement, "I feel more confident about assisting with an actual SSI/SSDI application having completed the practice case." As discussed below, a significant percentage of the evaluation period trainees registered for the SOAR OAT system and began documenting positive outcomes after completing the training.

Additionally, survey respondents were asked about the two things they liked the most and the least about the SOAR Online Course. The "most liked" features of the course question generated 200 comments from 102 respondents. The top two responses were the practice case component (23\%, 45 comments) and the comprehensive information in the course (16\%, 32 comments). Other well-liked features included the videos throughout the course and the feedback and support from the SOAR TA center.

The question asking what participants disliked the most about the course generated 146 comments from 90 respondents. Twenty comments were categorized as "not applicable" as they contained positive comments, "n/a," or "none." The top two responses after this indicated that the course is too long $(10 \%, 14$ comments) and that respondents did not like the online setting of the course $(9 \%$, 13 comments). Other disliked features of the course included confusion in how to get started with the course, writing a MSR, and too much commitment required to complete the training.

\section{Quantitative Results of SOAR Outcomes from Trainees}

During the base year, 151 trainees who successfully completed the course registered for the OAT system. Of these trainees, 64 entered outcomes for SSI/SSDI applications into the system. This $42 \%$ data entry rate is much higher than from those who received the in-person training during the evaluation period. By contrast, of the 210 individuals who received the in-person training and 
Content comprehension

1. I have a better understanding of the differences between SSI and SSDI, including the health insurance offered and eligibility requirements

2. I am better able to identify the non-medical criteria for SSI/SSDI eligibility

3. I have a better understanding of the disability determination process and how to develop medical evidence to support a disability

4. I have a clearer understanding of the role of functional information in the determination of disability

5. I feel more equipped to thoroughly interview an SSI/SSDI client and assess his/her functioning

6. I will be able to write a comprehensive medical summary to be submitted for disability determination

7. Overall, I feel this training will help me do a better job assisting individuals with SSI/SSDI applications

\section{Course content}

8. The course was comprehensive and the information was up-to-date

9. The course was interesting and held my attention

10. The course presented the information clearly

11. The course was organized in a way that was conducive to learning

12. The balance of information among written materials, videos, and the practice case was effective

13. The tools and worksheets reference in the course will be useful for my work in the field

\section{Practice case component}

14. The practice case helped me to better understand the material being covered in the course

15. Completing the practice case helped me explore how I will use the information in my own work

registered for the OAT system during the evaluation period, only 65 trainees (31\%) entered data into OAT.

Providers who took the online course documented outcomes on 405 initial applications for SSI/SSDI, with an average approval rate of $71 \%, 6 \%$ points higher than the national SOAR average approval rate of $65 \%$. Providers reported receiving the decisions in 91 days, compared to national averages of 94 and 81 days in 2014 and 2015, respectively. SOAR Online Course trainees also assisted with 116 appeals, which include reconsiderations and ALJ hearings. Sixty-one percent of these appeals were approved, which compares to a national average for SOAR-assisted appeals of $52 \%$.

Interestingly, the approval rates for both SOAR-assisted initial applications (71\%) and appeals (61\%) were the same for the group who received in-person training and registered for OAT during the base year. This group completed 495 initial claims and 146 appeals. The key difference was that this group averaged 109 days to decision for initial claims. However, it is unclear what role training modality had in this outcome, as differences could be due to varying decision times in the provider's regions.

\section{DISCUSSION AND IMPLICATIONS}

The SOAR Online Course was developed to expand the reach of SOAR training, ensure standardized curriculum, and increase the capacity for public health workers to assist with SSI/SSDI applications. This evaluation aimed to address five key research questions.

\section{What Types of Caseworkers Are Utilizing the SOAR Online Course?}

We found that the majority of trainees who successfully completed the course self-identified as case managers, while a minority identified as state, local, or agency leads. Combining the case manager role with peer specialists, direct service workers account for $55 \%$ of all successful completions. This figure corresponds to the general structure of social service roles, with more direct service providers than oversight roles. It also corresponds well with the marketing of the course. As the aim of the course is to provide training in direct SSI/SSDI application assistance, we gear marketing toward direct service providers. We provide additional materials that are aimed toward supervisors to gain buy-in for the program, but this focus in on review of the course rather than full completion of the practice case.

We found that $34 \%$ of users left the role item blank during registration. In order to better understand the intended use of enrolled participants, PRA modified the enrollment for the online curriculum to include a question indicating the user's intention with the curriculum for future evaluation. Enrollees entering the course after the base year will indicate whether they are going to take Class 1 as an overview, look at particular articles as a refresher training, fully complete the course, or just browse for additional information. This will provide greater detail about why some individuals are enrolling but not completing the course. Other online training programs would benefit from including these types of registration questions during program launch, to more fully understand trainee intent. 


\section{What Benefits and Barriers Can We Identify by Using a Practice Case Component in the Online Training?}

The practice case component was a unique addition to the course during the transition to online training from in-person curriculum. This evaluation found that this component introduced a number of barriers and benefits for the training.

The practice case component does not allow the training participant to passively participate as they might in an in-person didactic presentation. It requires additional time, concentration, and investment to complete the activity. This barrier causes participants to self-select out of full completion of the online training because they do not have the time, the need, or do not see the value in successful completion. The high attrition rate with the SOAR Online Course is in line with previous studies; however, an additional barrier we found leading to high dropout rates, is the inability of case workers to bill for their time while completing the course.

The survey of trainees supports these findings, with many of the negative comments about the course relating to the length, time commitment, and the need to write a four to six page report as part of the practice case. Other online training programs should take into account the need for organizational support and flexibility in billing practices when integrating a practice case component into the training. This support is critical in retaining those trainees who could benefit from the course and reduce disincentives for taking additional training in the social service sector.

While there are some barriers to including the practice case component in the online course, this evaluation revealed benefits to integrating these generative learning activities. Including a realistic practice case that tests comprehension and application of information provided in the training helps training administrators know whether participants have increased their knowledge and benefited from the course. Integrating individual review of practice case materials and extensive feedback and technical assistance from the SOAR TA center ensures that trainees must fully understand the SSI/SSDI application process before successfully completing the course. For those trainees who do not pass the course on the first attempt and need additional revisions to their packets, reviewers provide comprehensive guidance and explanations. This additional support from national experts was not previously found in the in-person training model and provides an additional benefit to trainees.

Results from the qualitative surveys and feedback from the base year survey indicate strong positive views of the utility of the SOAR Online Course. The majority of respondents in the base year survey had not assisted with SSI/SSDI applications prior to the training but indicated an increase in confidence to do so after completion of the course. As shown below, many trainees from the evaluation translated this training into practice with SSI/SSDI applications. This finding presents an opportunity to overcome the barrier that in-person trainees faced, previously leading to a low number of applications post-training.

\section{Does Facilitated Training (i.e., Cohorts and Follow-up) Increase Participation in the Training?}

When analyzing course completions by location, we found that successful course completions were concentrated in Florida, Virginia, California, and Nevada. The concentration in California and Florida may be explained by the high number of enrolled users in both states (465 and 638, respectively). Virginia and Nevada have relatively low numbers of enrolled users (179 and 153 , respectively); however, the local and state leads in those areas facilitated training groups that helped individuals through the course, leading to a higher completion rate relative to the number of enrolled users.

The course usage mapping showed a high correlation between those areas that have local SOAR leaders and successful online course completion. While evaluation of the causal effect of local facilitators on completions was outside the scope of this study, the results indicate a positive connection between the two factors. SOAR leaders are taught to facilitate groups of trainees through the course, provide technical assistance on the training and SOAR process, and use blended training methods of the online course with in-person follow-up. These factors, along with the fact that SOAR leaders play a key role in the marketing of the course locally, may account for results found during the mapping process.

This indicates that utilizing the training cohort model developed by PRA and having local SOAR leaders to facilitate training can be effective in increasing course completion rates. Additionally, the data mapping indicates that the course has a wide reach across the United States and its territories, indicating that information about the training is expanding to new areas; however, future work will focus on increasing course completions and building capacity in rural areas.

\section{Does Online Training Increase the Quality and Quantity of Submitted SSI/SSDI Applications Compared to In-Person Training?}

Our evaluation results indicated that a higher percentage of trainees entered data into the OAT system if they were trained through the SOAR Online Course, as opposed to the in-person training model (42 versus $31 \%$ ). While the sample sizes for both groups were relatively small (151 and 210, respectively), this indicates a positive trend that should be explored in future evaluations. One possible reason for the higher data entry rate may be the strong emphasis in the SOAR Online Course on tracking outcomes and the OAT system. This material is not part of the in-person training curriculum and dissemination of information about OAT is at the discretion of individual trainers.

We found that the approval rates for both initial applications and appeals were the same between the two training modalities. The in-person training group assisted with more applications than the online training group during this period; however, the small sample size in this aspect of the evaluation limits our ability to extrapolate these findings and assess any significant changes in quantity or quality of applications. One limitation of these data 
is that we were only able to evaluate outcome results that were entered into the OAT system. We do not have internal access to individual outcomes that are entered into state-level databases or homeless management information systems (HMIS).

\section{How Can These Results Be Expanded into Other Areas of Online Training for Social Services?}

Other factors to be considered when designing an online training program is the length of time it takes for trainees to complete the course, particularly compared to in-person trainings. We found that those who successfully completed the course did so in an average of 44 days. An exception to this is the state lead role, which has an average of 78 days to completion of the course. This may be attributed to the competing demands of state leads, as they are often leading other statewide initiatives, such as SAMHSA's Projects for Assistance in Transition from Homelessness (PATH).

Results from this evaluation indicate that trainees need more time to complete the course than advertised on the course website. The SOAR Online Course marketing materials estimate that trainees can complete the course in approximately $16 \mathrm{~h}$ over 30 days; however, this was insufficient for many users. The discrepancy between advertised length and actual length may attribute to some of these negative views about course length. While removing the practice case element may shorten the training time, this evaluation finds that the benefits of including this active learning component outweigh the barriers for those who can commit the time. However, other social service trainings may not require as extensive a practice case as the one utilized by the SOAR Online Course, due to the intensive nature of SSI/SSDI applications. As a result, other trainings could reduce the amount of training time required to meet the programmatic needs.

In analyzing the course video utilization, we found that the length of video had a strong, negative correlation to percentage of the video that was viewed. As a result, the longer videos in our training curriculum are less likely to be viewed in their entirety. Trainees who do not watch videos in full miss out on key elements of the course. The course video that received the highest percentage of view time, excluding the outlier male video \#1, was $3 \mathrm{~min}$ and $50 \mathrm{~s}$ in length. This indicates that when creating course, content videos should be no longer than $4 \mathrm{~min}$ each, with shorter videos receiving more complete views.

Some study limitations merit comment. The base year surveys were only sent to those who successfully passed the course. Future research designs including individuals who enrolled in the course, but did not complete the training, would provide a better

\section{REFERENCES}

1. U.S. Department of Housing and Urban Development. HUD's 2013 Continuum of Care Homeless Assistance Programs: Homeless Populations and Subpopulations. (2015). Available from: https://www.hudexchange.info/ resource/reportmanagement/published/CoC_PopSub_NatlTerrDC_2013. pdf

2. Schoeni R, Koegel P. Economic resources of the homeless: evidence from Los Angeles. Contemp Econ Policy (1998) 16(3):295-308. doi:10.111 1/j.1465-7287.1998.tb00520.x understanding of barriers to course completion. Additionally, the base year survey was delivered in October 2015 and may have had a response bias toward those who most recently completed the training. We addressed this potential response bias by issuing course surveys to trainees as soon as they successfully complete the training beginning in December 2015.

\section{CONCLUSION}

As shown above, our evaluation demonstrates that an online training course for social service providers can be effective in increasing capacity for assisting vulnerable individuals. The SOAR Online Course expands training opportunities for individuals to learn how to effectively assist with SSI/SSDI applications for individuals experiencing or at risk for homelessness.

This evaluation revealed that key success factors for online training models include the integration of a practice case component (or other generative learning activity), support from local facilitators, and feedback and technical assistance for trainees. Further evaluation is needed regarding reasons behind high attrition rates in the course and causal links between facilitation and successful course completion; however, results indicate the potential for other public health providers to utilize online training models to increase knowledge and capacity.

\section{AUTHOR CONTRIBUTIONS}

Both authors made substantial contribution to the design and concept of the evaluation, and drafted the manuscript under review. KL contributed toward background research, narrative information about the course, analysis from the pilot testing phase of the evaluation, and discussion of the findings. JE contributed to the analysis and interpretation of base year study data, and overall implications and discussion of the findings. Both authors give final approval for the draft to be published and agree to be accountable for all aspects of the work in ensuring that questions related to the accuracy or integrity of any part of the work are appropriately investigated and resolved.

\section{ACKNOWLEDGMENTS}

The authors would like to acknowledge the contributions of PRA staff, Brian Case and Suzy Sodergren, and PRA's technical consultant and software engineer, Matthew Lupfer. The SOAR TA center, operated by PRA, is funded by the Substance Abuse and Mental Health Services Administration, Contract \# 283-12-5802.

3. Rosenheck R, Dausey D, Frisman L, Kasprow W. Outcomes after initial receipt of social security benefits among homeless veterans with mental illness. Psychiatr Serv (2000) 51(2):1549-54. doi:10.1176/appi.ps.51.12.1549

4. Fawcett J. Social Security disability and the mentally ill. Psychiatr Ann (2002) 32(5):272. doi:10.3928/0048-5713-20020501-03

5. World Health Organization, Calouste Gulbenkian Foundation. Social Determinants of Mental Health. Geneva: World Health Organization (2014).

6. Buchholz JR, Malte CA, Calsyn DA, Baer JS, Nichol P, Kivlahan DR, et al. Associations of housing status with substance abuse treatment and service use 
outcomes among veterans. Psychiatr Serv (2010) 61(7):698-706. doi:10.1176/ appi.ps.61.7.698

7. General Accounting Office. Homelessness: Challenges to Using Mainstream Programs. Washington, DC: U.S. GAO (2000).

8. U.S. Social Security Administration, Office of Retirement and Disability Policy. Annual Statistical Report on the Social Security Disability Insurance Program, 2015. Washington, DC: SSA (2015). SSA Pub. No. 13-11827.

9. U.S. Social Security Administration, The Office of Disability Adjudication and Review (ODAR). Average Wait Time until Hearing Held Report for the Month of December 2015. (2015). Available from: https://www.ssa.gov/appeals/ DataSets/archive/01_FY2016/01_December_NetStat_Report.html

10. Rosen J, Hoey R, Steed T. Food stamps and SSI benefits: removing access challenges for homeless people. J Poverty Law Policy (2001):670-96.

11. Dennis DL, Lassiter M, Connelly W, Lupfer K. Helping adults who are homeless gain disability benefits: The SOAR program. Psychiatr Serv (2011) 62(11):1373-6. doi:10.1176/ps.62.11.pss6211_1373

12. SAMHSA SOAR TA Center. 2015 SOAR Outcomes Summary. Delmar, NY: Policy Research Associates, Inc. (2016).

13. Kauff JF, Clary E, Lupfer KS, Fischer PJ. An evaluation of SOAR: implementation and outcomes of an effort to improve access to SSI and SSDI. Psychiatr Serv (2016). doi:10.1176/appi.ps.201500247

14. Means B, Toyama Y, Murphy R, Bakia M, Jones K. Evaluation of Evidence-Based Practices in Online Learning: A Meta-Analysis and Review of Online Learning Studies. Washington, DC: US Department of Education (2009).

15. Schell G, Janicki TJ. Online course pedagogy and the constructivist learning model. J South Assoc Inf Syst (2013) 1(1):26-36. doi:10.3998/ jsais.11880084.0001.104
16. Gao T, Lehman JD. The effects of different levels of interaction on the achievement and motivational perceptions of college students in a Web-based learning environment. J Interact Learn Res (2003) 14(4):367-86.

17. Zhang D. Interactive multimedia-based e-learning: a study of effectiveness. Am JDistance Educ (2005) 19(3):149-62. doi:10.1207/ s15389286ajde1903_3

18. Ballew P, Castro S, Claus J, Kittur N, Brennan L, Brownson RC. Developing webbased training for public health practitioners: what can we learn from a review of five disciplines? Health Educ Res (2013) 28(2):276-87. doi:10.1093/her/cys098

19. Heinrichs WL, Youngblood P, Harter PM, Dev P. Simulation for team training and assessment: case studies of online training with virtual worlds. World J Surg (2008) 32(2):161-70. doi:10.1007/s00268-007-9354-2

20. Stone DM, Barber CW, Potter L. Public health training online: the national center for suicide prevention training. Am J Prev Med (2005) 29(5):247-51. doi:10.1016/j.amepre.2005.08.019

21. Park JH, Choi HJ. Factors influencing adult learners' decision to drop out or persist in online learning. Educ Technol Soc (2009) 12(4):207-17.

Conflict of Interest Statement: This research was conducted in the absence of any commercial or financial relationships that could be construed as a potential conflict of interest.

Copyright $\odot 2016$ Lupfer and Elder. This is an open-access article distributed under the terms of the Creative Commons Attribution License (CC BY). The use, distribution or reproduction in other forums is permitted, provided the original author(s) or licensor are credited and that the original publication in this journal is cited, in accordance with accepted academic practice. No use, distribution or reproduction is permitted which does not comply with these terms. 